Rev. Chil, Pediatr. 59 (4): 253-257, 1988

\title{
Bitolterol: un nuevo broncodilatador en pediatría
}

\author{
Dr. Mario Calvo G. ${ }^{1}$; E.M. Pannela Arellano $\mathrm{F}^{2}$; Sr. Ftancisco Marín $\mathrm{M}^{3}$ \\ Bitolterol: a new bronchodilator evaluated in pediatries
}

\begin{abstract}
Bitolterol is a beta 2 adrenergic drug recently introduced in this country. Fifteen children with bronchial asthma were submitted to four daily provocative exercise tests, in each case, before and after bitolterol and placebo. A beneficial effect in VEF 1 ( $\mathrm{p}<0.001)$ was obtained with bitolterol both as prophylaxis and as treatment of exercise induced bronchoconstriction. Bitolterol seems to be a safe and efficient drug, thus becoming a new and adequate alternative for the treatment of children with bronchial asthma,

(Key words: bronchial asthnia, prophilaxis, treatment, exercise, bitolterol.)
\end{abstract}

El uso profiláctico y terapéutico de las drogas betamiméticas en asma bronquial $(\mathrm{AB})$ ha adquirido un rol fundamental en el adecuado control de esta enfermedad ${ }^{1,2}$. Al mismo tiempo, la vía inhalatoria ha demostrado ser aquella que tiene mejor efecto clínico con los menores efectos secundarios $^{3,4}$,

Recientemente se ha incorporado en nuestro país el bitolterol, beta dos adrenérgico de uso inhalatorio. Es un medicamento aportado en forma proactiva que se transforma en colterol, la fracción molecular activa, al ser hidrolizado por las estearasas de los tejidos y de la sangre ${ }^{5-7}$. Una inhalación aporta $0,37 \mathrm{mg}$ de mesilato de bitolterol.

Con el propósito de estudiar la seguridad y eficacia de este medicamento en pediatría, lo

1. Instituto de Pediatría, Facultad de Medicina. Universided Austral de Chile.

2. Enfermera-Matrona, Servicio de Salud. Valdivia.

3. Biosstadístico. Departamento de Salud Salud Públjca, Facultad de Medicina. Lniversidad Austral de Chile. evaluamos en el tratamiento de los episodios obs. tructivos agudos y antes de la realización de pruebas de ejercicio en niños con asma ${ }^{B-11}$.

\section{MATERIAL Y METODO}

Tin 15 nỉ̄os con asma bronquial y pruebas de provocación por ejercicio positivas se evaluaron mediante un estudio de simple ciego, en dos series de dos pruebas cada una, las respuestas de la vía aérea a la inhalación de bitolterol y placebo, respectivamente, después de provocar obstrucción bromquial con ejercicio. Fn los mismos pacientes se estudiaron también las respucstas de la vía aérea al ejercicio después de la inhalación de bitolterol y placebo en condiciones basales. Entre cada une de las dos pruebas medió, al menos, un lapso de 24 horas.

La edad de los pacientes fluctuó entre 5 y 14 ( $\bar{x} 7,7$; DE 3) ẫos; 9 eran de sexo masculino, ninguno había recibido drogas bconcodilatadoras ni presentado signos clínicos de obstrucción bronquial en la semana precedente y en todos la función pulmonar basal era normal.

La función respiratoria se midió en un espirómetro Vitalograph $S \Theta$ con impresor integral de pruebas de función pulmonar. E.l parámetro elegido para las mediciones fue el $\mathrm{VEF}_{1}$ expresado en porcentaje de $V E F_{1}$ teórico para la edart, sexo y estatura $8,9,14$. 
Fil ejercicio consistió en una carrera fibre de 8 minutos de duración.

En todos los casos se solicitó el consentimiento in. formado de los pacientes, sus padres o cuidadores y se instruyó a los sujetos en el método de ejercicio y el uso de los inhaladores ${ }^{16-18}$.

Todos los pacientes fueron sometidos en forma consecutiva a las pruebas de bitolterol y placebo posteriores y precedentes a las provocaciones. Se hicieron registros de frecuencia cardíaca y $\mathrm{VEF}_{1}$ basales a $\operatorname{los} 2$ y 8 minutos de iniciado el esfuerzo y 5,15 y 30 minutos después de finalizado el mismo.

En las cuatro pruebas se registran los efectos indeseatles que pudiese provocar el medjcamento, especificamente temblor, cefalea, nerviosismo, taquicardia, palpitaciones, extrasístoles, malestar torácico, tos, irritación faríngea, broncoespasmo, vértigo y mareos. La información obtenida fue procesada en computador y para el estudio estadístico se empleó la dócima t para muestras pareadas.

\section{RESULTADOS}

La reducción del VEF fue de igual magnitud después del ejercicio en las pruebas realizadas antes de suministrar bitolterol y placebo, siendo, en ambos casos, significativamente menor el $V E F_{1}$ después del ejercicio que el basal. Después de administrar bitolterol, el $\mathrm{VEF}_{1}$ fue significati. vamente mejor que después del placebo a los 5 . 15 y 30 minutos posteriores a la provocación, alcanzando valores iguales o mayores que en el registro basal (tabla 1).

El efecto profiláctico del bitolterol en la prueba de provocación con ejercicio se hizo evidente al término de éste y se registró en todo el perio. do de registro, hasta 30 minutos después del esfuerzo (tabla 2).

Tabla 1

VEF, después del ejercicio y de la administración de bitolterol y placebo en el tratariento de la obstrucción bronquiat inducida por ejercicio

\begin{tabular}{|c|c|c|c|c|c|}
\hline \multirow[t]{2}{*}{ Situación } & \multicolumn{2}{|c|}{ Bitolterol } & \multicolumn{2}{|c|}{ Placebo } & \multirow{2}{*}{$\mathrm{p}$} \\
\hline & $V E F_{\lambda}$ & (DE) & $V E F_{t}$ & $(\mathrm{DE})$ & \\
\hline Basal & 101,7 & $(7,4)$ & 102,8 & $(7,8)$ & $\overline{\text { N.S. }}$ \\
\hline $\begin{array}{c}\text { Ejercicio } \\
8 \mathrm{~min}\end{array}$ & 71,1 & $(9.5)$ & 71,1 & $(9,7)$ & N.S. \\
\hline $\begin{array}{l}\text { Post-tratam } \\
1 \mathrm{~min}\end{array}$ & 73,2 & $(8,6)$ & 70,2 & $(8,6)$ & N.S. \\
\hline $5 \mathrm{~min}$ & 98,9 & $(8,1)$ & 73,2 & $(6,5)$ & $<0,001$ \\
\hline $15 \mathrm{~min}$ & 106,3 & $(7,9)$ & 75,2 & $(7,3)$ & $<0,001$ \\
\hline $30 \mathrm{~min}$ & 107,6 & $(7,1)$ & 80,2 & $(7,2)$ & $<0,001$ \\
\hline
\end{tabular}

Tabla 2

Efecto preventivo de bitolterol y placebo en la obstrucción bronquial inducida por ejercicio

\begin{tabular}{|c|c|c|c|c|c|}
\hline \multirow[t]{2}{*}{ Situación } & \multicolumn{2}{|c|}{ Bitolterol } & \multicolumn{2}{|c|}{ Placcbo } & \multirow{2}{*}{ p } \\
\hline & $\mathrm{VEF}_{1}$ & (DE) & $\mathrm{VEF}_{1}$ & (DE) & \\
\hline Basal & 102,1 & $(7,2)$ & 104,3 & $(7,5)$ & N.S. \\
\hline $\begin{array}{l}\text { Ejercicio } \\
2 \text { min }\end{array}$ & 108,5 & $(7,0)$ & 106,0 & $(6,2)$ & N.S. \\
\hline $8 \min$ & 106,3 & $(6,9)$ & 71,5 & $(9,9)$ & $<0,001$ \\
\hline $\begin{array}{l}\text { Post-tratamiento } \\
1 \mathrm{~min}\end{array}$ & 106,9 & $(6,9)$ & 71,8 & $(9,8)$ & $<0,001$ \\
\hline $5 \min$ & 107,0 & $(6,2)$ & 72,1 & $(9,5)$ & $<0,001$ \\
\hline $15 \mathrm{~min}$ & 107,7 & $(6,9)$ & 73,0 & $(9,5)$ & $<0,001$ \\
\hline $30 \mathrm{~min}$ & 106,5 & $(5,7)$ & 78,8 & $(9,1)$ & $<0,001$ \\
\hline
\end{tabular}


No se observó aumento de la frecuencia cardíaca basal ni de la frecuencia al término del esfuerzo al comparar bitolterol y placebo antes y al término del ejercicio; por el contrario, la frecuencia cardíaca fue significativamente menor a $\operatorname{los} 5,15$ y 30 minutos post-esfuerzo cuando se empleo bitolterol para controlar la obstrucción inducida y a los 15 y 30 minutos post-esfuerzo al usarlos preventivamente.

\section{DISCUSION}

El efecto broncodilatador del bitolterol ha sido descrito en publicaciones extranjeras desde el año 1976 y con posterioridad existen estudios clínicos en adultos ${ }^{5,6,19}$. Este medicamento aportado en forma proactiva es hidrolizado por estearasas de los tejidos, producjéndose un potente broncodilatador beta dos, el colterol. Su efecto principal a nivel pulmonar se debe a que el tejido pulmonar presenta niveles de estearasas significativamente más altas que el plasma o corazón, concentrándose en pulmón ${ }^{6,19}$.

Se ha reportado una acción más prolongada del bitolterol que los broncodilatadores beta dos adrenérgicos que actualmente conocemos, to cual se deberia a una hidrólisis gradual de la droga, como al hecho que por presentar un grupo metilo tendria una susceptibilidad menor a la acción de la mono amino oxidasa. Su única via de administración es la inhalatoria ${ }^{5,20}$.

En nuestra experiencia, el efecto broncodilatador del bitolterol fue muy destacable ya desde los cinco minutos después de su inhalación al aplicársele a los pacientes en crisis. Asimismo, el efecto preventivo fue objetivable al impedir la broncoconstricción inducida por el ejercicio a los ocho minutos de éste, sin alterar la broncodilatación inicial que se produce a los dos minutos de ejercicio. En todas estas situaciones clínicas, al comparar bitolterol con placebo, la diferencia fue altamente significativa (tablas 1 y 2 ).

Su tolerancia fue óptima y no existió rechazo a su inhalación. En ningún paciente se evidenció taquicardia, lo cual concuerda con la alta especificidad que presenta por el tejido pulmonar, descrita en la literatura ${ }^{5,7,19}$. Al contrario, existió menor frecuencia cardíaca en el grupo tratado con bitolterol, ya sea en erisis (tabla 3) o preventivo (tabla 4), lo cual interpretamos se debe a que el grupo que recibió placebo mantuvo frecuencia cardiaca alta por persistir obstruido, mientras que el grupo tratado con bitolterol al ceder su broncoobstrucción disminuyó más rápidamente la frecuencia cardíaca elevada alcanzada con el ejercicio.

Un paciente relató palpitaciones al usar bitolterol en forma preventiva y fue posterior al ejercicio, lo cual no impidió continuar normalmente la prueba. El mismo paciente lo había tolerado adecuadamente al recibirlo como tratamiento de su crisis. No existieron otros efectos indeseables.

Concluimos que el bitolterol es un broncadila. tador altamente efectivo para ser utilizado en pediatría. Es bien tolerado al ser usado por vía inhalatoria $y$ en las dosis usadas en este estudio. Su rapidez de acción y especificidad por los betareceptores bronquiales lo transforman en una nueva $y$ adecuada alternativa en el tratamiento de niños afectos de asma bronquial.

Tabla 3

Frecuencia cardiaca después de la administración de bitolterol y placebo en el tratamiento de la obstrucción bronquial inducida por ejercicio

\begin{tabular}{|c|c|c|c|}
\hline Situación & Bitolterol & Placebo & $p$ \\
\hline & cpm $\bar{x}(\mathbf{D E})$ & $\operatorname{cpm} \bar{\lambda}(\mathrm{DE})$ & \\
\hline Basal & $78,1 \quad(5,4)$ & $78,8 \quad(7,2)$ & N.S. \\
\hline $\begin{array}{l}\text { Ejercicio } \\
8 \mathrm{~min}\end{array}$ & $187,3 \quad(8,5)$ & $186,4 \quad(6,2)$ & N.S. \\
\hline $\begin{array}{l}\text { Post-ejercicio } \\
1 \mathrm{~min}\end{array}$ & $186,9 \quad(6,9)$ & $183,8 \quad(6,3)$ & N.S. \\
\hline $5 \mathrm{~min}$ & $112,0 \quad(9,8)$ & $148,0 \quad(9,6)$ & $<0,05$ \\
\hline $15 \mathrm{~min}$ & $80,8 \quad(6,5)$ & $134,7 \quad(8,4)$ & $<0,01$ \\
\hline $30 \mathrm{~min}$ & $78,4 \quad(3,9)$ & $116,4 \quad(9,8)$ & $<0,05$ \\
\hline
\end{tabular}


Tabla 4

Frecuencia cardíaca con bitolterol y placebo anministrados para prevenir la obstrucción bronquial inducida por ejercicio

\begin{tabular}{|c|c|c|c|}
\hline \multirow[t]{2}{*}{ Situaciôn } & Bitolterol & Placebo & \multirow{2}{*}{ p } \\
\hline & cpm $\bar{x}(\mathrm{DE})$ & $\operatorname{cpm} \bar{x}(\mathrm{DE})$ & \\
\hline Basal & $79.2 \quad(4,2)$ & $78,8 \quad(4,3)$ & N.S. \\
\hline $\begin{array}{c}\text { Ejercicio } \\
2 \mathrm{~min}\end{array}$ & $98,3 \quad(9,3)$ & $100,0 \quad(8,5)$ & N.S. \\
\hline $8 \mathrm{~min}$ & $184,4 \quad(4,7)$ & $185,9 \quad(6,1)$ & N.S. \\
\hline $\begin{array}{l}\text { Post-ejercicio } \\
1 \mathrm{~min}\end{array}$ & $182,5 \quad(4,9)$ & $184,9 \quad(6,2)$ & N.S. \\
\hline $5 \mathrm{~min}$ & $120,0 \quad(5.8)$ & $142,1 \quad(9,6)$ & N.S. \\
\hline $15 \min$ & $100, ? \quad(9,7)$ & $133,7 \quad(9,5)$ & $<0,05$ \\
\hline 30 mirr & $83,5 \quad(7,6)$ & $115,8 \quad(9,3)$ & $<0,05$ \\
\hline
\end{tabular}

\section{RESUMEN}

El bitolterol es un beta dos adrenérgico sobre cuyo empleo en pediatria no existe experiencia. Se realizaron estudios de simple ciego para comparar los efectos de placebo y bitolterol en el tratamiento de episodios agudos de obstrucción bronquial $y$ antes de realizar pruebas de esfuerzo en 15 nifros con asma bronquial. Se obtuvo una diferencia estadísticamente significativa $(p<0,001)$ al analizar bitolterol vs. placebo a $\operatorname{los} 5,15$ y 30 minutos post-tratamiento. Igual situación al usarlo en forma preventiva a los 8 minutos de ejercicio y al minuto 5,15 y $30 \mathrm{mi}$ nutos post-ejercicio. Concluimos que el bitolterol es seguro y eficaz, constituyendo una nueva y adecuada alternativa en el tratamiento de niños con asma bronquial.

\section{REFERENCIAS}

1. Undurraga A.: Broncodilatadores, En Galleguillos F. Ed. Asma Bronquial. Aspectos clínicos e inmunológicos. Santiago. Ed. Arancibia, 1976; 102109.

2. De Paula F., Casas F: Asma Bronquial. Pathos, 1984: 60: $30-44$.

3. Spector $S$ : Oral and inhaled drugs in asthma: A current reappraisal. Imm and Allergy Pract, 1983; 5: 63-69.

4. Shenfield (i., Paterson J.: Clinical assessment of bronchodilatador drugs delivered by aerosol. Thorax, 1973;28: 124-128.
5. Koss J., Mingo $T .:$ Bitolterol mesylate (WIN 32784) aerosol. A new long-acting bronchodilator with reduced chronotropic effects. Chest, 1980; $78: 283-287$.

6. Shargel L., Dorrbecker $S$., Levitt M.: Physiological disposition and metabolism of $\mathrm{N}$-t-butylarterenol and its di-p-toluate ester (bitolterol) in the rat. Drug Metab Dispos, 1976; 4: 65-71.

7. Petty T., Scoggin Ch., Rollins R., Repsher L.: Bitolterol compared to isoproterenol in adyanced chronic Obstructive Pulmonary Disease. Chest, $1984 ; 86 ; 404-408$.

8. Calpo M. Fernández M., Oehling A.: Airway resistance and lung function in exercise-induced asthma. Allergol et Immunopathol., 1985; 13: 45-52.

9. Pienson W., Bieman C.: Free running test for exercise-induced bronchospasm. Pediatrics, 1975: 56 (Suppl); 890-892.

10. Hayness $R$. Ingram $R$, Mc Fadden E.: An assessment of the pulmonary response to exercise in asthma and an analysis of the factors influencing it. Am Rev Respir Dis, 1976; 114: 739-742.

11. Godfrey S., Silverman M., Anderson S.: Problems of interpreting exercise-induced asthma. I Allergy Clin Immunol, 1973; 52: 199-209.

12. American Thoracic Society. Definitions and classifications of thronic bronchitis, asthma and pulmonary emphysema. Am Rev Respir Dis 1962: 85: 761-768.

13. Godfrey $S_{*}$ : Fxercise-induced asthma, Allergy, 1978; 33: 229-237.

14. Jones $R$.: (Fd.) Función respiratoria. En Asma Infantil, Barcelona, Ed. Salvat, 1978; 80-92.

15. Prandi F.: (Ed.) Exploración de la función pulmonar. Progresos en asmatologia infantil. Barcelona, Ed. Sándoz, 1982; 60-72. 
16. Anderson S.: Connolly N., Gadfrey S.: Comparison of bronchoconstriction induced by cycling and ruming. Thorax, 1971;26: 396-401.

17. Jones R., Buston $M$., Wharton $M$.: The effects of exercise on ventilatory function in the child with asthma. Br $J$ Dis Chest, 1962; 56: 78-86.

18. Sly R.: Effect of B-adrenoreceptor stimulants on exercise-induced asthma. Pediatrics, 1975; 56 (Suppl): $910-915$.
19. Minatoya $H$.: Studies on bitolterol, di-p-toluate ester of N-tert-butylarterenol: a new long-acting bronchodilator with reduced cardiovascular effects. J Pharmacol Exp Ther, 1978: 206: 515-527.

20. Person H. Olsson $T$.: Some pharnacological properties of terbutalina, 1-(3,5 dehydroxyphenil)2-(t-butylamino)-ethanol: a new sympathomimetic B-receptor stimulating agent. Acta Med Scand, $1970 ; 512$ (Suppl): $11-18$. 